\title{
INNER GRADINGS AND GALOIS EXTENSIONS WITH NORMAL BASIS
}

\author{
MARGARET BEATTIE
}

(Communicated by Donald S. Passman)

\begin{abstract}
We prove that for $G$ a finite group, a $G$-graded Azumaya algebra over a commutative ring has inner grading if and only if an associated Galois extension has normal basis.
\end{abstract}

\section{INTRODUCTION}

In this note, we prove the following Skolem-Noether type theorem for graded Azumaya algebras over a commutative ring $k$. If $G$ is a finite group and $A$ a $G$-graded Azumaya algebra over $k$, then the $G$-grading on $A$ is inner if and only if the Galois extension $\left(A \#(k G)^{*}\right)^{A}$ has normal basis. In particular, for $k$ semilocal or von Neumann regular, every $G$-grading of a $k$-Azumaya algebra is inner. This result extends the work of Osterburg and Quinn [8] who proved a similar result for $k$ a field and $A$ strongly $G$-graded.

Our main theorem parallels the well-known situation for $A$ an Azumaya $k G$-module algebra. For an automorphism $\Omega$ of $A$ is inner if and only if $A(\Omega)=\{x \in A: a x=x \Omega(a)$ for all $a \in A\}$ is a free $k$-module of rank 1 , and thus $k G$ acts innerly on $A$ if and only if the Galois $k G$-object $\bigoplus_{\sigma \in G} A(\sigma)$ has normal basis.

\section{Preliminaries}

Throughout, $k$ will denote a (trivially graded) commutative ring with 1 and $G$ a finite group. Unless otherwise specified, $\otimes$, Hom, etc. should be understood to be over $k$, algebras and modules are $k$-algebras and $k$-modules, etc.

The Hopf algebra $(k G)^{*}$ is the dual of the group ring $k G ;(k G)^{*}$ has a free basis over $k$, namely the projections $p_{\sigma}, \sigma \in G$, where $p_{\sigma}(\tau)=\delta_{\sigma, \tau}$. Thus the $p_{\sigma}$ are a set of orthogonal idempotents with $\sum_{\sigma \in G} p_{\sigma}=1$.

A $k$-algebra $A$ is called a $G$-graded algebra if $A=\bigoplus_{\sigma \in G} A_{\sigma}, A_{\sigma} A_{\tau} \subseteq A_{\sigma \tau}$ and $k \subseteq A_{1} . A$ is a $G$-graded algebra if and only if $A$ is a $(k G)^{*}$-module

Received by the editors June 20, 1988 and, in revised form, December 14, 1988. Presented to the 849th meeting of the AMS, May 19-20, 1989.

1980 Mathematics Subject Classification (1985 Revision). Primary 16A03, 16A16, 16 A74.

This research was partially supported by Natural Sciences and Engineering Research Council of Canada grant \#A9137. 
algebra. Thus we may form the smash product $A \#(k G)^{*}$ where $A \#(k G)^{*}=$ $A \otimes(k G)^{*}$ as a $k$-module and has multiplication defined by $\left(a \# p_{\sigma}\right)\left(b \# p_{\tau}\right)=$ $a b_{\sigma \tau^{-1}} \# p_{\tau}, b_{r}$, being the $\gamma$-th homogeneous component of $b \in A$. $G$ acts as a group of automorphisms on $A \#(k G)^{*}$ by $\sigma\left(a \# p_{\tau}\right)=a \# p_{\tau \sigma^{-1}} ;\left(A \#(k G)^{*}\right)^{G}=$ $A$. (See [5] for further details of this construction.)

A $(k G)^{*}$-module algebra $A$ is said to have inner grading if there are maps $u$ and $"$ from $G$ to $A$ such that for all $a \in A, \gamma \in G$,

$$
a_{\gamma}=\sum_{r \in G} u(\gamma \alpha) a_{u}\left(\alpha^{-1}\right) .
$$

Then, since $1 \in A_{1}$,

$$
\sum_{r \in G} u(\gamma \alpha) \mu\left(\alpha^{-1}\right)=\delta_{i, 1} .
$$

It was noted in [8] that for $A$ finite dimensional over a field $k$, we also have

$$
\sum_{r \in G} u(\gamma \alpha) u\left(\alpha^{-1}\right)=\delta_{i, 1} .
$$

The equation (2) holds also for $A$ finitely generated projective over $k, k$ a commutative ring. Equation (1) says that $u$ is right invertible in the convolution algebra $C=\operatorname{Hom}\left((k G)^{*}, A\right)$ (see [10, Chapter IV] for the definition of $C$ ). But then $m_{1 /}$, multiplication on the right by $u$, is a $k$-module isomorphism from $C$ to $C u$, so that, at each localization of $k, C$ and $C u$ are free of the same rank. Thus, the identity map from $C u$ to $C$ is an isomorphism at each localization, and thus is an isomorphism. Then $C=C u$ and $u$ is left invertible in $C$. Since left and right inverses are equal, (2) holds.

Recall that a (not necessarily commutative) $k$-algebra $S$ is called a Galois extension of $k$ with group $G$ or a Galois $(k G)^{*}$-object [3] if $G$ acts as a group of automorphisms on $S$ such that $S^{G}=k$ and the map $\Gamma: S \otimes S \rightarrow S \otimes(k G)^{*}$ defined by $\Gamma(s \otimes t)=\sum_{\sigma \in G} s \sigma(t) \otimes p_{\sigma}$ is an $S G$-module isomorphism where the $G$-action on $S \otimes S$ is induced by the $G$-action on the second factor and the $G$-action on $S \otimes(k G)^{*}$ is induced by the usual $G$-action on $(k G)^{*}$.

A Galois extension $S$ is said to have normal basis if $S$ is isomorphic to $(k G)^{*}$ as $(k G)^{*}$-comodules (or, equivalently, as $k G$-modules). $S$ has normal basis if and only if there exists $x \in S$ such that $\{\sigma(x): \sigma \in G\}$ is a free basis for the $k$-module $S$.

Note that the definition of an inner grading comes from Sweedler's more general definition of an inner Hopf algebra action [9]. This more general setting for Skolem-Noether type results is studied in [2] for $k$ a field; if the Hopf algebra $H$ is $(k G)^{*}$, the results of [8] are recovered. The method of proof in $[2,8]$ and in this paper are closely related. In fact, using [4, 3.4], one easily sees that for $A$ strongly $G$-graded, the Galois extension $E$ of [8] and the Galois 
extension $S(A)$ described below are isomorphic Galois extensions of $k$ with group $G$.

\section{THE MAIN THEOREM}

From now on, $A$ will denote a finitely generated projective central separable $G$-graded algebra. We define a Galois extension $S(A)$, associated with $A$, as follows. The algebra $A \#(k G)^{*}$ has a left $A \otimes A^{o}$-module structure defined by $a \otimes b^{o} \circ x=a \# 1 \circ x \circ b \# 1$. We form $S(A)=\left(A \#(k G)^{*}\right)^{A}=\left\{x \in A \#(k G)^{*}:\right.$ $a \otimes 1^{\circ} \circ x=1 \otimes a^{o} \circ x$ for all $\left.a \in A\right\}$, i.e. $S(A)$ is the centralizer of the subalgebra $A$ in $A \#(k G)^{*}$. It was shown in [1, p. 688] that $S(A)$ is a Galois extension for $G$ abelian. The proof also holds for nonabelian $G$; we include it here for the sake of completeness.

Since $S(A)^{G}=\left(A \#(k G)^{*}\right)^{G} \cap S(A)=A^{A}=k$, it remains to show that the map $\Gamma: S(A) \otimes S(A) \rightarrow S(A) \otimes(k G)^{*}$ is an isomorphism. Note that since $A$ is an Azumaya algebra and $A \#(k G)^{*}$ is a left $A \otimes A^{o}$-module, the map $m: A \otimes S(A)=$ $A \otimes\left(A \#(k G)^{*}\right)^{A} \rightarrow A \#(k G)^{*}$ defined by $m(a \otimes x)=a x$ is an isomorphism [7, 2.13]. It is easy to check that the map $\eta:\left(A \#(k G)^{*}\right) \otimes_{A}\left(A \#(k G)^{*}\right) \rightarrow$ $\left(A \#(k G)^{*}\right) \otimes(k G)^{*}$ defined by $\eta\left(\left(a \# p_{\sigma}\right) \otimes_{A}\left(b \# p_{\tau}\right)\right)=\sum_{\alpha \in G}\left(a b_{\alpha} \# p_{\alpha \alpha^{-1} \sigma}\right) \otimes p_{\sigma^{-1} \alpha \tau}$ is bijective with inverse $\eta^{-1}$ defined by $\eta^{-1}\left(\left(a \# p_{\sigma}\right) \otimes p_{\tau}\right)=\left(a \# p_{\sigma}\right) \otimes_{A}\left(1 \# p_{\sigma \tau}\right)$. The following diagram commutes (cf. [4, p. 310]).

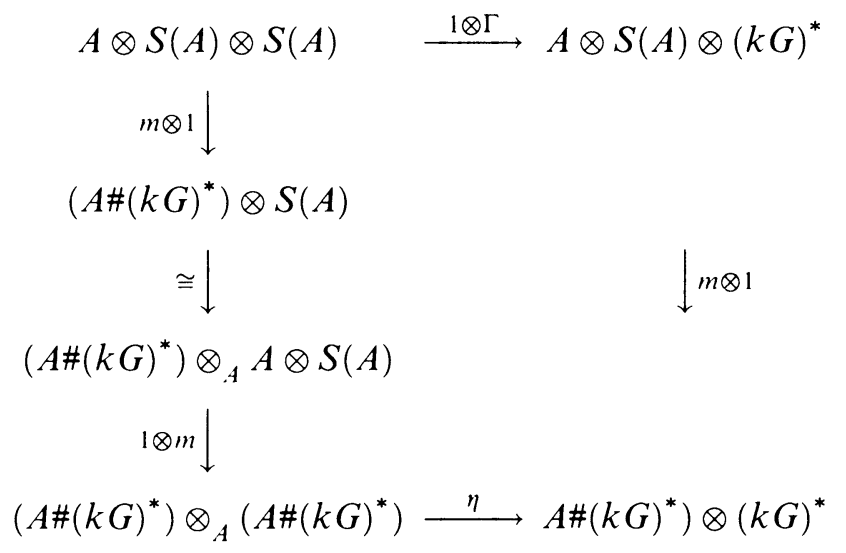

Since all the maps but $1 \otimes \Gamma$ are already known to be isomorphisms, $1 \otimes \Gamma$ is an isomorphism and since $A$ is faithfully flat, $\Gamma$ is also. Thus, $S(A)$ is indeed a Galois extension with group $G$.

Theorem. The following statements are equivalent.

(i) The G-grading on $A$ is inner.

(ii) The Galois extension $S(A) \supseteq k$ has a normal basis.

(iii) There exist $x, y \in S(A)$ such that $\sum_{\sigma \in G} \sigma(x) \tau \sigma(y)=\delta_{\tau, 1}$.

Proof. We show that (i) $\Rightarrow$ (ii) $\Rightarrow$ (iii) $\Rightarrow$ (i).

(i) $\Rightarrow$ (ii). Suppose first that the $G$-grading on $A$ is inner, i.e. there exist 
maps $u$ and $a$ from $G$ to $A$ such that for all $a \in A, \gamma \in G, a_{\gamma}=$ $\sum_{\alpha \in G} u(\gamma \alpha) a u\left(\alpha^{-1}\right)$. Define a $k$-module map $\Omega$ from $(k G)^{*}$ to $S(A)$ by $\Omega\left(\beta^{-1}\left(p_{1}\right)\right)=\Omega\left(p_{\beta}\right)=\sum_{\alpha \in G} \mu\left(\alpha^{-1}\right) \# p_{\alpha \beta}=\beta^{-1}\left(\Omega\left(p_{1}\right)\right)$. Note that $\Omega\left(p_{1}\right)$, and therefore the image of $\Omega$, is in $S(A)=\left(A \#(k G)^{*}\right)^{A}$ since for $c \in A$,

$$
\begin{aligned}
\left(\sum_{\alpha \in G} a\left(\alpha^{-1}\right) \# p_{\alpha}\right)(c \# 1) & =\sum_{\alpha, \tau \in G} a\left(\alpha^{-1}\right) c_{\alpha \tau^{-1}} \# p_{\tau} \\
& =\sum_{\alpha, \tau, \sigma \in G} u\left(\alpha^{-1}\right) \mu\left(\alpha \tau^{-1} \sigma\right) c u\left(\sigma^{-1}\right) \# p_{\tau} \\
& =\sum_{\sigma, \tau \in G}\left\{\sum_{\alpha \in G} u\left(\alpha^{-1}\right) \mu\left(\alpha \tau^{-1} \sigma\right)\right\} c u\left(\sigma^{-1}\right) \# p_{\tau} \\
& =\sum_{\sigma, \tau \in G}\left\{\delta_{1, \tau^{-1} \sigma}\right\} c u\left(\sigma^{-1}\right) \# p_{\tau} \quad \text { by }(2) \\
& =\sum_{\tau \in G} c u\left(\tau^{-1}\right) \# p_{\tau} \\
& =(c \# 1) \sum_{\alpha \in G} u\left(\alpha^{-1}\right) \# p_{\alpha} .
\end{aligned}
$$

Therefore for all $c \in A$,

$$
\operatorname{cu}(\gamma)=\sum_{\beta \in G} u\left(\beta^{-1}\right) c_{\beta \gamma}=\sum_{\delta \in G} u\left(\gamma \delta^{-1}\right) c_{\delta} .
$$

It remains to show that $\Omega$ is an isomorphism.

Suppose $\Omega\left(\sum_{\sigma \in G} r(\sigma) p_{\sigma}\right)=\sum_{\sigma, \alpha \in G} r(\sigma) \mu\left(\sigma \alpha^{-1}\right) \# p_{\alpha \alpha}=0$ for scalars $r(\sigma) \in$ $k, \sigma \in G$. Then $\sum_{\sigma \in G} r(\sigma) \alpha\left(\sigma \alpha^{-1}\right)=0$ for all $\alpha \in G$. Then, for any $\lambda \in G$,

$$
0=\sum_{\sigma \in G} r(\sigma) \mu\left(\sigma \alpha^{-1}\right) \varkappa\left(\alpha \lambda^{-1}\right),
$$

so that

$$
\begin{aligned}
0 & =\sum_{\kappa \in G} \sum_{\sigma \in G} r(\sigma) \mu\left(\sigma \alpha^{-1}\right) \mu\left(\alpha \lambda^{-1}\right) \\
& =\sum_{\sigma \in G} r(\sigma)\left\{\sum_{\rho \in G} u\left(\sigma \lambda^{-1} \rho^{-1}\right) \mu(\rho)\right\} \quad \text { where } \rho=\alpha \lambda^{-1} \\
& =r(\lambda) \quad \text { by }(2) .
\end{aligned}
$$

Thus $\Omega$ is one-one. Locally $\Omega$ is an isomorphism since locally $S(A)$ is free of rank equal to the order of $G$; therefore $\Omega$ is an isomorphism.

(ii) $\Rightarrow$ (iii). This implication follows from [8, Lemma 2]. 
(iii) $\Rightarrow($ i). We suppose next that there exist $x, y \in S(A)$ such that $\sum_{\sigma \in G} \sigma(x) \tau \sigma(y)=\delta_{\tau, 1}$. Let $x=\sum_{\alpha \in G} a(\alpha) \# p_{\alpha}, y=\sum_{\beta \in G} b(\beta) \# p_{\beta}$. Then

$$
\begin{aligned}
\delta_{\tau, 1} & =\sum_{\sigma \in G} \sigma(x) \tau \sigma(y) \\
& =\sum_{\sigma, \beta \in G}(\sigma(x))\left(b(\beta) \# p_{\beta(\tau \sigma)^{-1}}\right) \\
& =\sum_{\sigma, \beta \in G}(b(\beta) \# 1) \sigma(x)\left(1 \# p_{\beta(\tau \sigma)^{-1}}\right) \quad \text { since } \sigma(x) \in S(A) \\
& =\sum_{\alpha, \beta, \sigma \in G}\left(b(\beta) a(\alpha) \# p_{\left(\alpha \sigma^{-1}\right.}\right)\left(1 \# p_{\beta(\tau \sigma)^{-1}}\right) \\
& =\sum_{\beta, \sigma \in G} b(\beta) a\left(\beta(\tau \sigma)^{-1} \sigma\right) \# p_{\beta(\tau \sigma)^{-1}} \\
& =\sum_{\beta, \lambda \in G} b(\beta) a\left(\lambda \tau^{-1} \lambda^{-1} \beta\right) \# p_{\lambda} \quad \text { with } \lambda=\beta(\tau \sigma)^{-1}
\end{aligned}
$$

and since the coefficient of $p_{\lambda}$ is $\delta_{\tau, 1}$ for all $\lambda$, we have

$$
\sum_{\beta \in G} b(\beta) a(\eta \beta)=\delta_{\eta, 1} \quad \text { for all } \eta \in G .
$$

Now for any $c \in A$,

$$
\begin{aligned}
c_{\tau} & =\sum_{\gamma \in G}\left\{\delta_{\tau, \gamma}\right\} c_{\gamma} \\
& =\sum_{\gamma \in G}\left\{\sum_{\rho \in G} b(\tau \rho) a(\gamma \rho)\right\} c_{\gamma} \quad \text { by }(* *) \\
& =\sum_{\rho \in G} b(\tau \rho)\left\{\sum_{\gamma \in G} a(\gamma \rho) c_{\gamma}\right\} \\
& =\sum_{\rho \in G} b(\tau \rho) c a(\rho) \quad \text { since } x \in S(A) .
\end{aligned}
$$

Now define maps $u, u$ from $G$ to $A$ by $u(\sigma)=b(\sigma)$ and $u\left(\tau^{-1}\right)=a(\tau)$.

This completes the proof of the main theorem. Note that in the proof that (iii) $\Rightarrow(\mathrm{i})$, the fact that $y$ is in $S(A)$ is not used. In fact, condition (iii) is equivalent to the following:

(iii) $^{\prime}$ There exist $x \in S(A), y \in A \#(k G)^{*}$ such that $\sum_{\sigma \in G} \sigma(x) \tau \sigma(y)=\delta_{\tau, 1}$.

Corollary 1. If $\operatorname{MSpec}(k)$, the maximal ideal spectrum of $k$, has a basis of open/closed sets, (for example if $k$ is semilocal or von Neumann regular), then every $G$-graded $k$-Azumaya algebra has inner grading.

Proof. It is proved in [6] that if $\operatorname{MSpec}(k)$ has a basis of open/closed sets, and $H$ is a finitely generated commutative or cocommutative Hopf algebra, 
then every Galois $H$-object has normal basis. Let $H$ be the commutative Hopf algebra $(k G)^{*}$.

Corollary 2. If every G-graded $k$-Azumaya algebra has inner grading, then every $(k G)^{*}$-Galois object has normal basis.

Proof. The statement follows from the fact that every Galois $(k G)^{*}$-object is isomorphic to $S(A)$ for some graded Azumaya algebra $A$. The proof is essentially the same as $[1$, p. 689-90]. We outline the argument.

If $S$ is a Galois extension of $k$ with group $G$, then $A=S \# k G$ is a $G$-graded Azumaya algebra with $A_{\sigma}=S \# \sigma, \sigma \in G$. Then

$$
\phi: S(A)=\left((S \# k G) \#(k G)^{*}\right)^{(S \# k G)} \rightarrow S
$$

defined by $\phi\left(\sum_{\sigma, \tau \in G}(s(\sigma, \tau) \# \sigma) \# p_{\tau}\right)=\sum_{\sigma \in G} s(\sigma, 1)$ is a $k G$-algebra homomorphism. But a $k G$-algebra homomorphism between two Galois extensions is an isomorphism.

\section{ACKNOWLEDGMENT}

I would like to acknowledge the referee's helpful comments.

\section{REFERENCES}

1. M. Beattie, A direct sum decomposition for the Brauer group of $H$-module algebras, J. Alg. $\mathbf{4 3}$ (1976), 686-693.

2. R. J. Blattner and S. Montgomery, Crossed products and Galois extensions of Hopf algebras, Pacific J. Math. 137 (1989), 37-54.

3. S. U. Chase and M. E. Sweedler, Hopf algebras and Galois theory, Lecture Notes in Math. 97, Springer-Verlag, Berlin, 1969.

4. L. N. Childs, G. Garfinkel and M. Orzech, The Brauer group of graded Azumaya algebras, Trans. Amer. Math. Soc. 175 (1973), 299-325.

5. M. Cohen and S. Montgomery, Group graded rings, smash products, and group actions, Trans. Amer. Math. Soc. 282 (1984), 237-258.

6. H. F. Kreimer and P. M. Cook II, Galois theories and normal bases, J. Alg. 43 (1976), 115-121.

7. M. Orzech and C. Small, The Brauer group of commutative rings, Lecture Notes in Pure and Appl. Math., No. 11, Marcel Dekker, New York, 1975.

8. J. Osterburg and D. Quinn, A Noether Skolem theorem for group-graded rings, J. Alg. 113 (1988), 483-490; Addendum, J. Alg. 120 (1989), 414-415.

9. M. E. Sweedler, Cohomology of algebras over Hopf algebras, Trans. Amer. Math. Soc. 133 (1968), 205-239.

10. __ Hopf algebras, Benjamin, New York, 1969.

Department of Mathematics and Computer Science, Mount Allison University, Sackville, New Brunswick, Canada E0A 3C0 